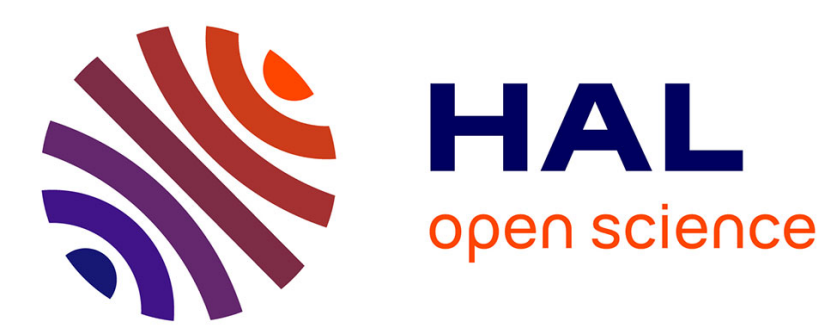

\title{
Delayed nonlocal reaction-diffusion model for hematopoietic stem cell dynamics with Dirichlet boundary conditions
}

\author{
Mostafa Adimy, Abdennasser Chekroun, Toshikazu Kuniya
}

\section{To cite this version:}

Mostafa Adimy, Abdennasser Chekroun, Toshikazu Kuniya. Delayed nonlocal reaction-diffusion model for hematopoietic stem cell dynamics with Dirichlet boundary conditions. Mathematical Modelling of Natural Phenomena, 2017, 12 (6), pp.1 - 22. 10.1051/mmnp/2017078 . hal-01683636

\section{HAL Id: hal-01683636 \\ https://hal.inria.fr/hal-01683636}

Submitted on 14 Jan 2018

HAL is a multi-disciplinary open access archive for the deposit and dissemination of scientific research documents, whether they are published or not. The documents may come from teaching and research institutions in France or abroad, or from public or private research centers.
L'archive ouverte pluridisciplinaire HAL, est destinée au dépôt et à la diffusion de documents scientifiques de niveau recherche, publiés ou non, émanant des établissements d'enseignement et de recherche français ou étrangers, des laboratoires publics ou privés. 


\title{
DELAYED NONLOCAL REACTION-DIFFUSION MODEL FOR HEMATOPOIETIC STEM CELL DYNAMICS WITH DIRICHLET BOUNDARY CONDITIONS
}

\author{
M. Adimy ${ }^{1}$, A. Chekroun ${ }^{2}$ And T. Kuniya ${ }^{3}$
}

\begin{abstract}
The paper focuses on the mathematical analysis and modeling of hematopoietic stem cell (HSC) dynamics that lead to the production and regulation of blood cells in the bone morrow. The HSC population is seen as a continuous medium structured in age and space. Using the method of characteristics, we reduce the age structured system to a reaction-diffusion equation containing a nonlocal spatial term and a time delay. Firstly, we give some properties on the existence, uniqueness and positivity of the solution. Secondly, we obtain a threshold condition for the global asymptotic stability of the trivial steady state by using a Lyapunov functional and we prove that if it is not globally asymptotic stable then, it is unstable. Thirdly, we give sufficient conditions for the existence and uniqueness of the positive steady state by using the sub- and super-solutions method. Finally, we prove the uniform persistence of the system when the trivial steady state is unstable. Throughout the paper, we provide some numerical simulations to illustrate our results.
\end{abstract}

Mathematics Subject Classification. 34B18, 35B35, 37N25, 92C37.

Received October 30, 2017. Accepted November 13, 2017.

\section{INTRODUCTION}

All blood cells arise from a common origin in the bone marrow: the hematopoietic stem cells (HSCs). These stem cells are undifferentiated and have a high proliferative potential. They can proliferate and mature to form all types of blood cells: the red blood cells, white cells and platelets [33]. Hematopoiesis is the term used to describe this process of production and regulation of blood cells. The HSCs have abilities to produce by division either similar cells with the same maturity level (self-renewal), or cells committed to one of the three blood cell types (differentiation). Each HSC can be either in a proliferating phase or in a quiescent phase (also called $G_{0}$-phase or resting phase). Proliferating cells are in the cell cycle where they are committed to divide during mitosis at the end of this phase. After division, each cell gives birth to two daughter cells which enter immediately the quiescent phase [8, 17]. Quiescent cells represent the main part of the HSC population $(90 \%$ of HSCs are in a quiescent compartment [8]).

It is believed that several hematological diseases are due to some abnormalities in the feedback loops between circulation blood cells and HSC population (see [2, 5, 20, 21] and the references therein). Among a wide variety of

Keywords and phrases: Age-space-structured PDE, time-delayed reaction-diffusion equation, Dirichlet boundary conditions, existence and uniqueness of positive steady state, Lyapunov functional, cell dynamics.

${ }^{1}$ Inria, Université de Lyon, Université Lyon 1, Institut Camille Jordan, 43 Bd. du 11 novembre 1918, 69200 Villeurbanne Cedex, France. mostafa.adimy@inria.fr

${ }^{2}$ Laboratoire d'Analyse Nonlinéaire et Mathématiques Appliquées, Université de Tlemcen, Tlemcen 13000, Algeria.

${ }^{3}$ Graduate School of System Informatics, Kobe University, 1-1 Rokkodai-cho, Nada-ku, Kobe 657-8501, Japan. 
disorders affecting blood cells, myeloproliferative diseases are of great interest. They are characterized by a group of conditions that cause blood cells to grow abnormally. They include chronic myelogenous leukemia, a cancer of white blood cells. Myeloproliferative disorders usually originate from the HSC compartment: an uncontrolled proliferation in the HSC compartment can perturb the entire system and leads to a quick proliferation [33].

Mathematical modeling of HSC dynamics has been introduced to our best knowledge by Mackey [20], in 1978. It is based on earlier works by Lajtha [17] and Burns and Tannock [8]. The model of Mackey is an uncoupled system of two nonlinear delay differential equations which considers: proliferating and quiescent HSCs. The delay describes the average cell cycle duration. The model of Mackey stressed the influence of some factors such as the apoptotic rate and the introduction rate, the cell cycle duration, playing an important role in the appearance of periodic solutions. Since then, Mackey's model has been improved by many other authors (see $[1-6,21,24,25]$ and the references therein).

Almost all of the previous works are based on delayed differential systems without space. They neglect the effects of spatial structure on the cell population dynamics. However, we know that the diffusion of cells can play an important role in determining collective behavior. The bone marrow environment plays an essential role in the regulation of HSCs by providing specialized niches in which these cells are maintained [33]. In particular, in many myeloproliferative disorders, the excessive proliferation of malignant HSCs changes normal spacial cell distribution in the bone marrow. If proliferation of malignant HSCs is sufficiently fast, then the disease can invade the whole bone marrow.

In this paper, we propose and analyze a mathematical model of HSC dynamics by taking into account the spacial diffusion of HSCs in the bone marrow (considered as a bounded domain with homogeneous Dirichlet boundary conditions). Only few models have been developed to study hematopoiesis with diffusion (for instance, see $[4,7,10,18])$. In $[4,18]$, the authors considered a model of HSC dynamics with diffusion in unbounded domain. They showed the existence of traveling wave fronts with a minimal wave speed that coincides with the asymptotic speed of spread for solutions. In [7], a multi-scale model of erythropoiesis (the part of hematopoiesis that produces red blood cells) was introduced. It is based on ordinary differential equations (for intracellular network that regulates the behavior of each cell), reaction-diffusion system (for extracellular proteins as growth factors that act as signaling between cells) and individual based model (for the cell population). In [10], the authors described the evolution of leukemic HSCs in the bone marrow with a reaction-diffusion-convection system in porous medium.

In another side, there have been extensive investigations on nonlocal and time-delayed population models in order to study the effects of spatial diffusion and time delay on the behavior of population (see Gourley and Wu's survey paper [13] and references therein). It is known that the stability analysis of the delayed reaction-diffusion equations in a bounded domain can be obtained by analyzing the sign of the real part of characteristic equation's roots (see [34]) or by finding the principal eigenvalue (see [37]). In recent years, the stability analysis and the existence of periodic solutions and their direction for delayed reaction-diffusion systems have been widely studied by many authors such as in $[9,11,15,16,19,22,23,26-29,31,32,35,36,38]$ where in particular, Hopf bifurcations were proved to occur with Dirichlet or Neumann boundary conditions.

In the next section, we first propose the model for HSC dynamics which takes the form of an age-spacestructured system with diffusion in one dimensional bounded domain (with homogeneous Dirichlet boundary conditions). We reduce the age-structured system to a time-delayed reaction-diffusion HSC model with nonlocal spatial term. In Section 3, the existence and uniqueness of solutions for the reduced delayed reaction-diffusion system are studied. Section 4 is devoted to the study of stability of the trivial steady state. By using a Lyapunov functional, we give a condition for the trivial steady state to be globally asymptotically stable. In the case when it is not globally stable, we prove its instability. In Section 5, we establish under additional conditions the existence and uniqueness of a positive steady state. In Section 6, the uniform (strong) persistence of the system when the trivial steady state is unstable is established. Some numerical simulations are also carried out to illustrate our results. 


\section{BIOLOGICAL BACKGROUND AND THE MODEL}

We investigate a nonlinear age-space-structured system. We will see that this system can be reduced by using the method of characteristics to a reaction-diffusion system with a nonlocal spatial term and a time delay.

As mentioned before, the HSCs can be either quiescent (represented by the density $n$ ) or proliferating (represented by the density $p$ ). We consider structured variability in the both compartments at time $t \geq 0$, age $a \geq 0$ and position $x \in \Omega$. The age represents the time spent by a cell in a compartment (quiescent or proliferating). For the space, we take $x$ in a bounded one dimensional domain $\left(l_{1}, l_{2}\right)$. The diffusion rates of cells are denoted $d_{1}, d_{2}>0$ for the quiescent and proliferating cells, respectively. The proliferating cells die by apoptosis with a rate $\gamma \geq 0$ and divide at age $a=\tau$ in two daughter cells, which enter directly the quiescent phase. The quiescent cells can differentiate with a rate $\delta \geq 0$ or be introduced in the proliferating phase with a rate $\beta$ (see [20]). We assume that the rate $\beta$ is continuously differentiable, positive, and depends upon the total density of resting cells (see [20]), denoted by $N(t, x)$, where

$$
N(t, x)=\int_{0}^{+\infty} n(t, x, a) \mathrm{d} a .
$$

Moreover, $\beta$ is supposed to be a decreasing function with $\lim _{N \rightarrow+\infty} \beta(N)=0$.

Example 2.1. In previous works (see $[20,24,25]$ ), the authors considered $\beta$ as a Hill function given by

$$
\beta(N)=\frac{\beta_{0} \theta^{r}}{\theta^{r}+N^{r}}, \quad r>1
$$

where $\beta_{0} \geq 0$ is the maximal rate of transition between quiescent phase and proliferating phase, $\theta$ is the value for which $\beta$ attains half of its maximum value, and $r$ is the sensitivity of the rate of reintroduction. It describes the reaction of $\beta$ due to external stimuli, the action of a growth factor for example (some growth factors are known to trigger the introduction of quiescent cells in the proliferating phase).

We describe the evolution of the HSC population by the following partial differential system, for $t>0$ and $x \in\left(l_{1}, l_{2}\right)$,

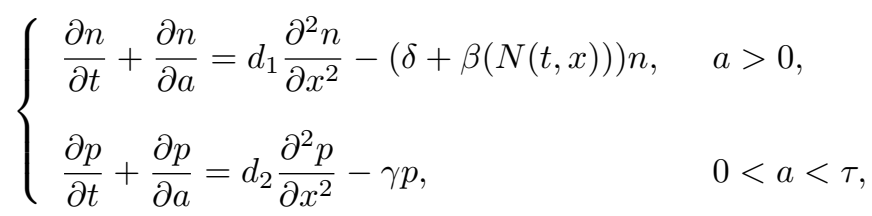

where $\tau$ is the duration of the proliferating phase. This system is completed by age-boundary conditions, for $t>0$ and $x \in\left(l_{1}, l_{2}\right)$,

$$
\left\{\begin{array}{l}
n(t, x, 0)=2 p(t, x, \tau), \\
p(t, x, 0)=\beta(N(t, x)) N(t, x),
\end{array}\right.
$$

and initial conditions, for $x \in\left(l_{1}, l_{2}\right)$,

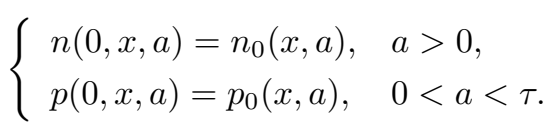


We also consider the homogeneous Dirichlet boundary conditions, for $t>0$,

$$
\begin{cases}n\left(t, l_{1}, a\right)=n\left(t, l_{2}, a\right)=0, & a>0 \\ p\left(t, l_{1}, a\right)=p\left(t, l_{2}, a\right)=0, & 0<a<\tau .\end{cases}
$$

The first equation of (2.3) describes the new quiescent cells and the coefficient 2 represents the division of each mother cell. The second equation is the flux of cells from quiescent phase to the proliferating one with a rate $\beta$ over all age $a \in[0,+\infty)$. The model is completed by the following reasonable condition:

$$
\lim _{a \rightarrow+\infty} n(t, x, a)=0, \quad t>0, x \in\left(l_{1}, l_{2}\right) .
$$

Without loss of generality, we use the following change of variables $x \mapsto \pi\left(x-l_{1}\right) /\left(l_{2}-l_{1}\right)$. Then, the domain becomes $(0, \pi)$ and the rates of diffusion become $d_{1}\left(\pi /\left(l_{2}-l_{1}\right)\right)^{2}$ and $d_{2}\left(\pi /\left(l_{2}-l_{1}\right)\right)^{2}$ (we denote them again $d_{1}$ and $\left.d_{2}\right)$. Moreover, the null Dirichlet boundary conditions are now imposed for $x=0$ and $x=\pi$.

We reduce the system (2.2) by integrating the first equation over the age variable and by using the method of characteristics for the second equation. We obtain

$$
\frac{\partial N(t, x)}{\partial t}=d_{1} \frac{\partial^{2} N(t, x)}{\partial x^{2}}-(\delta+\beta(N(t, x))) N(t, x)+2 p(t, x, \tau)
$$

We can solve the second equation of (2.2). Then, the expression of $p$ is given explicitly by

$$
p(t, x, \tau)= \begin{cases}e^{-\gamma t} \int_{0}^{\pi} \Gamma_{2}(t, x, s) p(0, s, \tau-t) \mathrm{d} s, & (t, x) \in[0, \tau] \times[0, \pi], \\ e^{-\gamma \tau} \int_{0}^{\pi} \Gamma_{2}(\tau, x, s) p(t-\tau, s, 0) \mathrm{d} s, & (t, x) \in(\tau,+\infty) \times[0, \pi],\end{cases}
$$

where $\Gamma_{2}$ is the Green's function associated to $d_{2} \Delta$ with Dirichlet boundary conditions. It is given by the following expression:

$$
\Gamma_{2}(t, x, s)=\frac{2}{\pi} \sum_{k=1}^{+\infty} e^{-k^{2} d_{2} t} \sin (k s) \sin (k x)
$$

For $d_{2}>0$, the function $\Gamma_{2}$ satisfies the following estimation (the proof is established in [14])

$$
0<\Gamma_{2}(\tau, x, s)<\frac{1}{\pi}\left(\frac{2}{e^{d_{2} \tau}-1}\right), \quad \text { for all } x, s \in(0, \pi) .
$$

We are interested in the long time behavior, so we can suppose $t>\tau$. The case where $t \in[0, \tau]$ can be treated to get an initial condition on an interval of length $\tau$. Then, a translation in time allows us to define a function $\phi$ such that $N(\theta, x)=\phi(\theta, x)$ for $\theta \in[-\tau, 0]$ and $x \in[0, \pi]$. Finally, we obtain the following system, for $x \in(0, \pi)$,

$$
\left\{\begin{array}{rlrl}
\frac{\partial N(t, x)}{\partial t}= & d_{1} \frac{\partial^{2} N(t, x)}{\partial x^{2}}-(\delta+\beta(N(t, x))) N(t, x) & \\
& +2 e^{-\gamma \tau} \int_{0}^{\pi} \Gamma_{2}(\tau, x, s) \beta(N(t-\tau, s)) N(t-\tau, s) \mathrm{d} s, & & t>0, \\
N(t, 0)=N(t, \pi)=0, & & t>0, \\
N(t, x)=\phi(t, x), & & t \in[-\tau, 0] .
\end{array}\right.
$$


The existence and uniqueness of solutions for (2.7) is given in the next section by using the theory of abstract functional differential equations (see [34]). First, we establish the positivity of the solutions of (2.7).

Theorem 2.2. Let $\phi(\theta, x)$, for $(\theta, x) \in[-\tau, 0] \times[0, \pi]$, be a nonnegative initial condition of $(2.7)$ and $N(t, x)$ be the corresponding solution. Then, $N$ is nonnegative.

Proof. We have $N(\theta, x) \geq 0$ for $(\theta, x) \in[-\tau, 0] \times[0, \pi]$. We assume that $[0, b)$ is the interval of existence of $N$ for some $b>0$. We proceed by steps on

$$
[0, b)=[0, \tau) \cup[\tau, 2 \tau) \cup \cdots \cup[n \tau, b),
$$

where $n=\lfloor b / \tau\rfloor$ is the integer part of $b / \tau$. Let take $t \in[0, \tau)$, which implies that $t-\tau \in[-\tau, 0)$. Then, $N(t-\tau, x)=\phi(t-\tau, x)$ and the equation (2.7) becomes

$$
\begin{aligned}
& \frac{\partial N(t, x)}{\partial t}-d_{1} \frac{\partial^{2} N(t, x)}{\partial x^{2}}+(\delta+\beta(N(t, x))) N(t, x) \\
& \quad=2 e^{-\gamma \tau} \int_{0}^{\pi} \Gamma_{2}(\tau, x, s) \beta(\phi(t-\tau, s)) \phi(t-\tau, s) \mathrm{d} s \geq 0 .
\end{aligned}
$$

We put $U(t, x)=e^{-\varrho t} N(t, x)$ and $f(t, x)=\delta+\beta(N(t, x))+\varrho$, for $\varrho>0$. So, the above equation deals to

$$
\begin{cases}\frac{\partial U(t, x)}{\mathrm{d} t}-d_{1} \frac{\partial^{2} U(t, x)}{\partial x^{2}}+f(t, x) U(t, x) \geq 0, & (t, x) \in[0, \tau) \times[0, \pi], \\ U(0, x)=\phi(0, x) \geq 0, & x \in[0, \pi] .\end{cases}
$$

Using the boundedness of $\beta$ and taking $\varrho>0$ so large to ensure the positivity of $f(t, x) \geq 0$, the maximum principle implies that $U(t, x) \geq 0$ for $(t, x) \in[0, \tau) \times[0, \pi]$. That means $N(t, x) \geq 0$ for $(t, x) \in[0, \tau) \times[0, \pi]$. We repeat the same argument in $[k \tau,(k+1) \tau)$, for $k=1,2, \ldots, n$. Then, we obtain

$$
N(t, x) \geq 0, \quad(t, x) \in[0, b) \times[0, \pi] .
$$

The proof is completed.

Remark 2.3. If the initial condition is continuous and satisfies $\phi(\theta, x)>0$, for all $\theta \in[-\tau, 0]$ and some $x \in(0, \pi)$, then $N$ is positive. This can be seen from the strict inequality in the proof of Theorem 2.2.

\section{EXISTENCE AND UNIQUENESS OF SOLUTIONS}

The existence and uniqueness of solutions for time-delayed reaction-diffusion equations are usually established using the theory of functional differential equations. The results obtained in this section are known in the literature (see [34]).

Consider $\mathbb{X}=C([0, \pi], \mathbb{R})$ the space of continuous functions with the classical supremum norm $\|u\|_{\mathbb{X}}=$ $\max _{s \in[0, \pi]}|u(s)|$ and the positive cone $\mathbb{X}^{+}=\{u \in \mathbb{X}: u(x) \geq 0, x \in[0, \pi]\}$. We have $\mathbb{X}$ as an ordered Banach space with respect to the cone $\mathbb{X}^{+}$. It is well known that the differential operator $A:=d_{1} \Delta$ with the domain $D(\Delta)=\left\{v \in C^{2}([0, \pi], \mathbb{R}) ; v(0)=v(\pi)=0\right\}$ generates a $C^{0}$-semigroup $(T(t))_{t>0}$ on $\mathbb{X}$, which is also analytic (see [34]). The standard parabolic maximum principle (see Corollary 7.2.3 of [26]) implies that the semigroup $T(t): \mathbb{X} \rightarrow \mathbb{X}$ is strongly positive. That is to say $T(t)\left(\mathbb{X}^{+} \backslash\{0\}\right) \subset \operatorname{Int}\left(\mathbb{X}^{+}\right), t>0$, where $\operatorname{Int}\left(\mathbb{X}^{+}\right)$is the interior of $\mathbb{X}^{+}$.

Let $\mathbb{Y}=C([-\tau, 0], \mathbb{X})$ and $\mathbb{Y}^{+}=C\left([-\tau, 0], \mathbb{X}^{+}\right)$. We can identify an element $u \in \mathbb{Y}$ as a function from $[-\tau, 0] \times$ $[0, \pi]$ into $\mathbb{R}$ by $u(\theta)(x)=u(\theta, x)$. For a continuous function $u:[-\tau, b) \rightarrow \mathbb{X}$, with $b>0$ and $t \in[0, b)$ we define 
$u_{t} \in \mathbb{Y}$ by $u_{t}(\theta)=u(t+\theta), \theta \in[-\tau, 0]$. We consider the function $F: \mathbb{Y}^{+} \rightarrow \mathbb{X}$ defined, for $\phi \in \mathbb{Y}^{+}$and $x \in[0, \pi]$, by

$$
F(\phi)(x)=-(\delta+\beta(\phi(0)(x))) \phi(0)(x)+2 e^{-\gamma \tau} \int_{0}^{\pi} \Gamma_{2}(\tau, x, s) \beta(\phi(-\tau)(s)) \phi(-\tau)(s) \mathrm{d} s .
$$

We write the system (2.7) in an abstract form

$$
\left\{\begin{array}{l}
\frac{\mathrm{d} u(t)}{\mathrm{d} t}=A u(t)+F\left(u_{t}\right), \quad t>0 \\
u_{0}=\phi \in \mathbb{Y}^{+} .
\end{array}\right.
$$

An integral representation of this system, for $t>0$, is

$$
u(t)=T(t) \phi(0)+\int_{0}^{t} T(t-s) F\left(u_{s}\right) \mathrm{d} s
$$

with $u(t)=\phi(t)$, for $t \in[-\tau, 0]$. The solutions of (3.2) are called mild (or integral) solutions of (3.1). We define the distance between an element $u \in \mathbb{X}$ and a set $A \subset \mathbb{X}$ by

$$
\operatorname{dist}(u, A)=\inf \left\{\|u-\zeta\|_{\mathbb{X}}: \zeta \in A\right\} .
$$

We have, for all $\phi \in \mathbb{Y}^{+}$,

$$
\lim _{h \rightarrow 0^{+}} \operatorname{dist}\left(\phi(0)+h F(\phi), \mathbb{X}^{+}\right)=0 .
$$

Moreover, $T(t): \mathbb{X}^{+} \rightarrow \mathbb{X}^{+}$. Then, we conclude from [34] the following result.

Theorem 3.1. Let $\phi \in \mathbb{Y}^{+}$. Then, equation (2.7) has a unique mild solution $N \in \mathbb{X}^{+}$defined on an interval $[-\tau, b)$, with $b>0$. Moreover, it is classical (strong) solution for $t>\tau$.

Proof. Consequence of Corollary 1.3, page 270 and Corollary 2.2.5, page 50 of [34] (see also Prop. 3 and Rem. 2.4 of $[22])$.

The global existence of solution of system (2.7) can be easily proven by the comparison with a linear problem and by the fact that the function $N \mapsto \beta(N)$ is monotone decreasing.

\section{Instability AND GLOBAL ATTRACTIVITY OF THE TRIVIAL STEADY STATE}

In this section, our main result concerns the stability of the trivial steady state. We start by giving a necessary condition for its instability. We consider the linearization of system (2.7) around the trivial steady state

$$
\left\{\begin{aligned}
\frac{\partial N(t, x)}{\partial t}= & d_{1} \frac{\partial^{2} N(t, x)}{\partial x^{2}}-(\delta+\beta(0)) N(t, x) \\
& +2 e^{-\gamma \tau} \beta(0) \int_{0}^{\pi} \Gamma_{2}(\tau, x, s) N(t-\tau, s) \mathrm{d} s \\
N(t, 0)= & N(t, \pi)=0 .
\end{aligned}\right.
$$


The corresponding nonlocal eigenvalue problem is given (see [37], p. 223) by

$$
\left\{\begin{aligned}
\lambda v(x)= & d_{1} \frac{\mathrm{d}^{2} v(x)}{\mathrm{d} x^{2}}-(\delta+\beta(0)) v(x) \\
& +2 e^{-\gamma \tau} \beta(0) \int_{0}^{\pi} \Gamma_{2}(\tau, x, s) v(s) \mathrm{d} s \cdot e^{-\lambda \tau}, \quad x \in(0, \pi), \\
v(0)= & v(\pi)=0 .
\end{aligned}\right.
$$

As $2 e^{-\gamma \tau} \beta(0)>0$, it follows from [37], Theorem 9.2.1, that problem (4.2) has a principal eigenvalue that has the same sign as the principal eigenvalue of the problem

$$
\left\{\begin{aligned}
\lambda v(x)= & d_{1} \frac{\mathrm{d}^{2} v(x)}{\mathrm{d} x^{2}}-(\delta+\beta(0)) v(x) \\
& +2 e^{-\gamma \tau} \beta(0) \int_{0}^{\pi} \Gamma_{2}(\tau, x, s) v(s) \mathrm{d} s, \quad x \in(0, \pi) \\
v(0)= & v(\pi)=0 .
\end{aligned}\right.
$$

One can remark that

$$
\int_{0}^{\pi} \Gamma_{2}(t, x, s) \sin (s) \mathrm{d} s=e^{-d_{2} t} \sin (x), \quad x \in(0, \pi), t>0
$$

Then, the principal eigenvalue of (4.3) is

$$
\lambda_{0}=-d_{1}-\delta-\beta(0)+2 e^{-\gamma \tau-d_{2} \tau} \beta(0) \in \mathbb{R}
$$

and it is associated to a strictly positive eigenvector

$$
v(x)=\sin x, \quad x \in(0, \pi) .
$$

Then, following the sign of $\lambda_{0}$, we have the local asymptotic stability and the instability of the trivial steady state. More precisely we have the following result:

Proposition 4.1. 1. Assume that

$$
d_{1}+\delta+\beta(0)>2 e^{-\gamma \tau-d_{2} \tau} \beta(0)
$$

Then, the zero steady state of (2.7) is locally asymptotically stable.

2. Assume that

$$
d_{1}+\delta+\beta(0)<2 e^{-\gamma \tau-d_{2} \tau} \beta(0)
$$

Then, it is unstable.

In fact, under the condition (4.4), we have the global asymptotic stability of the trivial steady state. This result is given by the following theorem.

Theorem 4.2. Assume that the condition (4.4) is satisfied. Then, the zero steady state of (2.7) is global asymptotic stable. 
Proof. We define a distance function in $\mathbb{X}($ see $[30])$

$$
d(u, w):=\int_{0}^{\pi} v(x)|u(x)-w(x)| \mathrm{d} x, \quad u, w \in \mathbb{X}
$$

where $v(x)=\sin (x), x \in(0, \pi)$. We proceed to construct a Lyapunov functional, $V: \mathbb{Y}^{+} \rightarrow \mathbb{R}^{+}$, along the solutions of (2.7),

$$
V\left(N_{t}\right):=\int_{0}^{\pi} v(x)\left(N(t, x)+\int_{t-\tau}^{t} 2 e^{-\gamma \tau} \int_{0}^{\pi} \Gamma_{2}(\tau, x, s) \beta(N(u, s)) N(u, s) \mathrm{d} s \mathrm{~d} u\right) \mathrm{d} x .
$$

Since $v(x)=\sin (x)>0$ on $(0, \pi), V$ is nonnegative, and $V(\varphi)=0, \varphi \in \mathbb{Y}^{+}$if and only if $\varphi \equiv 0$. Furthermore, for $N_{t} \in \mathbb{Y}^{+}$,

$$
V\left(N_{t}\right) \geq \int_{0}^{\pi} v(x) N(t, x) \mathrm{d} x=\mathrm{d}(N(t, \cdot), 0)
$$

Then, along the solution trajectory, we have

$$
\begin{aligned}
\dot{V}\left(N_{t}\right)= & \int_{0}^{\pi} v(x)\left(\frac{\partial N(t, x)}{\partial t}+2 e^{-\gamma \tau} \int_{0}^{\pi} \Gamma_{2}(\tau, x, s) \beta(N(t, s)) N(t, s) \mathrm{d} s\right. \\
& \left.-2 e^{-\gamma \tau} \int_{0}^{\pi} \Gamma_{2}(\tau, x, s) \beta(N(t-\tau, s)) N(t-\tau, s) \mathrm{d} s\right) \mathrm{d} x, \\
= & \int_{0}^{\pi} v(x)\left(d_{1} \frac{\partial^{2} N(t, x)}{\partial x^{2}}-(\delta+\beta(N(t, x))) N(t, x)\right. \\
& \left.+2 e^{-\gamma \tau} \int_{0}^{\pi} \Gamma_{2}(\tau, x, s) \beta(N(t, s)) N(t, s) \mathrm{d} s\right) \mathrm{d} x .
\end{aligned}
$$

Using the integration by parts formula, we have

$$
\begin{aligned}
\int_{0}^{\pi} v(x) \frac{\partial^{2} N(t, x)}{\partial x^{2}} \mathrm{~d} x & =\left[v(x) \frac{\partial N(t, x)}{\partial x}\right]_{0}^{\pi}-\int_{0}^{\pi} \frac{\mathrm{d} v(x)}{\mathrm{d} x} \frac{\partial N(t, x)}{\partial x} \mathrm{~d} x \\
& =-\left(\left[\frac{\mathrm{d} v(x)}{\mathrm{d} x} N(t, x)\right]_{0}^{\pi}-\int_{0}^{\pi} \frac{\mathrm{d}^{2} v(x)}{\mathrm{d} x^{2}} N(t, x) \mathrm{d} x\right) \\
& =\int_{0}^{\pi} \frac{\mathrm{d}^{2} v(x)}{\mathrm{d} x^{2}} N(t, x) \mathrm{d} x
\end{aligned}
$$

So, using (4.3), we obtain

$$
\begin{aligned}
\int_{0}^{\pi} v(x) d_{1} \frac{\partial^{2} N(t, x)}{\partial x^{2}} \mathrm{~d} x= & \int_{0}^{\pi}\left[\lambda_{0} v(x)+(\delta+\beta(0)) v(x)\right. \\
& \left.-2 e^{-\gamma \tau} \beta(0) \int_{0}^{\pi} \Gamma_{2}(\tau, x, s) v(s) \mathrm{d} s\right] N(t, x) \mathrm{d} x \\
= & \left(\lambda_{0}+\delta+\beta(0)-2 e^{-\gamma \tau-d_{2} \tau} \beta(0)\right) \int_{0}^{\pi} v(x) N(t, x) \mathrm{d} x .
\end{aligned}
$$


On the other hand, recalling that for $s, x \in(0, \pi)$,

$$
\Gamma_{2}(\tau, x, s)=\frac{2}{\pi} \sum_{k=1}^{+\infty} e^{-k^{2} d_{2} \tau} \sin (k s) \sin (k x)=\Gamma_{2}(\tau, s, x) .
$$

Then, by changing the order of integration we obtain

$$
\begin{aligned}
\int_{0}^{\pi} v( & x) \int_{0}^{\pi} \Gamma_{2}(\tau, x, s) \beta(N(t, s)) N(t, s) \mathrm{d} s \mathrm{~d} x \\
= & \int_{0}^{\pi} \beta(N(t, s)) N(t, s) \int_{0}^{\pi} \Gamma_{2}(\tau, s, x) v(x) \mathrm{d} x \mathrm{~d} s \\
= & \int_{0}^{\pi} e^{-d_{2} \tau} \beta(N(t, s)) N(t, s) v(s) \mathrm{d} s .
\end{aligned}
$$

Hence, substituting (4.9) and (4.10) into (4.7), we get

$$
\begin{aligned}
\dot{V}\left(N_{t}\right)= & \int_{0}^{\pi} v(x) N(t, x)\left[\lambda_{0}+\delta+\beta(0)-2 e^{-\gamma \tau-d_{2} \tau} \beta(0)\right. \\
& \left.-(\delta+\beta(N(t, x)))+2 e^{-\gamma \tau-d_{2} \tau} \beta(N(t, x))\right] \mathrm{d} x, \\
= & \int_{0}^{\pi} v(x) N(t, x)\left[\lambda_{0}+\left(1-2 e^{-\gamma \tau-d_{2} \tau}\right)(\beta(0)-\beta(N(t, x)))\right] \mathrm{d} x .
\end{aligned}
$$

Note that $\beta(0)-\beta(N(t, x)) \geq 0$ since $\beta(N)$ is a decreasing function. We consider two cases:

1. If $1-2 e^{-\gamma \tau-d_{2} \tau} \leq 0$, then we have

$$
\dot{V}\left(N_{t}\right) \leq \lambda_{0} \int_{0}^{\pi} v(x) N(t, x) \mathrm{d} x \leq 0
$$

since $\lambda_{0}=-d_{1}-\delta-\beta(0)+2 e^{-\gamma \tau-d_{2} \tau} \beta(0)<0$ under (4.4).

2. If $1-2 e^{-\gamma \tau-d_{2} \tau}>0$, then we have

$$
\begin{aligned}
\dot{V}\left(N_{t}\right) & \leq \int_{0}^{\pi} v(x) N(t, x)\left(\lambda_{0}+\left(1-2 e^{-\gamma \tau-d_{2} \tau}\right) \beta(0)\right) \mathrm{d} x, \\
& =-\left(d_{1}+\delta\right) \int_{0}^{\pi} v(x) N(t, x) \mathrm{d} x \leq 0 .
\end{aligned}
$$

Consequently, we get

$$
\dot{V}\left(N_{t}\right) \leq-\underline{\delta} d(N(t, \cdot), 0) \leq 0
$$

where $\underline{\delta}:=\min \left\{-\lambda_{0}, d_{1}+\delta\right\}>0$. Now, we define the notations $\gamma(\phi):=\cup_{t \geq 0}\left\{N(t, \cdot) \in \mathbb{X}^{+}\right\}$to denote the positive orbit of $(2.7)$ corresponding to the initial condition $\phi \in \mathbb{Y}^{+}$and $B_{r}(u):=\{w \in \mathbb{X}: d(w, u)<r\}$ to denote the open ball in $\mathbb{X}$ centered at $u$ with radius $r>0$. From above, we see that $V$ is a Lyapunov function. Then, from ([30], Thm. 1.1 in Chap. IV), we have

$$
V\left(N_{t}\right) \leq V(\phi)+\int_{0}^{t} \dot{V}\left(N_{s}\right) \mathrm{d} s, \quad t>0 .
$$




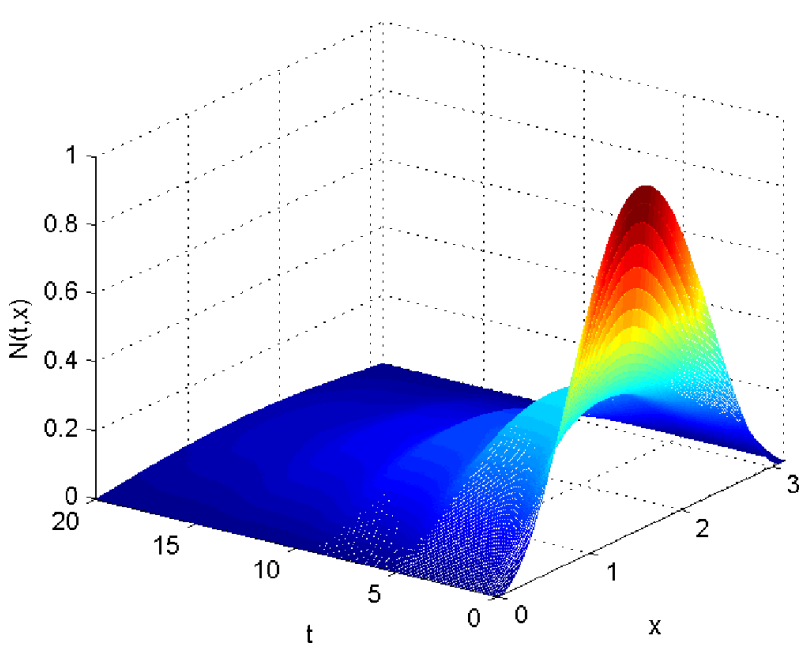

Figure 1. The global asymptotic stability of the trivial steady state of (2.7) is shown in this figure with $\beta$ a Hill function (2.1). The parameters are $: \delta=0.05, \beta_{0}=1.77, \theta=1, \gamma=0.2$, $\tau=4, r=5, d_{1}=0.1$ and $d_{2}=0.1$. The initial condition is $N(\theta, x)=(1-\cos (2 x)) / 2$, for $x \in(0, \pi)$ and $\theta \in[-\tau, 0]$.

Since $V$ is nonincreasing, we see that $V\left(N_{t}\right) \rightarrow \inf _{s \geq 0} V\left(N_{s}\right)=: \alpha \in \mathbb{R}^{+}$as $t \rightarrow+\infty$. Suppose by contradiction that $\alpha>0$. In this case, we have that $\gamma(\phi) \cap \cup_{t \geq 0}\left\{N(t, \cdot) \in \mathbb{X}^{+}: V\left(N_{t}\right)<\alpha\right\}$ is empty. From the continuity of $V$, we see that there exists a sufficiently small $\nu>0$ such that $\gamma(\phi) \cap B_{\nu}(0)$ is empty. Then, from (4.11), we have

$$
0 \leq V\left(N_{t}\right) \leq V(\phi)-\int_{0}^{t} \underline{\delta} d(N(s, \cdot), 0) \mathrm{d} s \leq V(\phi)-\underline{\delta} \nu t \rightarrow-\infty \quad \text { as } t \rightarrow+\infty,
$$

which is a contradiction. Therefore, $\alpha=0$. It follows from (4.6) that

$$
d(N(t, \cdot), 0) \rightarrow 0 \quad \text { as } t \rightarrow+\infty .
$$

This implies the global asymptotic stability of the trivial steady state (Fig. 1).

\section{EXISTENCE AND UNIQUENESS OF POSITIVE STEADY STATE}

The positive steady states of the partial differential equation (2.7) are the positive solutions which are stationary in time. They can be non-uniformly in space. Such solutions play an important role in the study of the dynamics of reaction-diffusion equations arising from biology. The local or global convergence of solutions to a positive steady state requires first the existence of this steady state. A positive steady state, denoted by $N(x)$, is a solution of the following stationary problem:

$$
\left\{\begin{aligned}
&-d_{1} \frac{\mathrm{d}^{2} N(x)}{\mathrm{d} x^{2}}=-(\delta+\beta(N(x))) N(x) \\
&+2 e^{-\gamma \tau} \int_{0}^{\pi} \Gamma_{2}(\tau, x, s) \beta(N(s)) N(s) \mathrm{d} s, \quad x \in(0, \pi), \\
& N(0)=N(\pi)=0 .
\end{aligned}\right.
$$


Our approach is based on the monotone iteration technique, where sub- and super-solutions are founded. Let

$$
H(N)(x):=d_{1} \frac{\mathrm{d}^{2} N(x)}{\mathrm{d} x^{2}}-(\delta+\beta(N(x))) N(x)+2 e^{-\gamma \tau} \int_{0}^{\pi} \Gamma_{2}(\tau, x, s) \beta(N(s)) N(s) \mathrm{d} s
$$

Then, we look for a function $x \mapsto N(x)$ which satisfies $H(N)(x)=0$. We define a sub-solution $x \mapsto \underline{N}(x)$ and a super-solution $x \mapsto \bar{N}(x)$ by

$$
\begin{aligned}
& H(\underline{N})(x) \geq 0 \\
& H(\bar{N})(x) \leq 0 \\
& 0<\underline{N}(x) \leq \bar{N}(x) \quad \text { on } x \in(0, \pi), \\
& \underline{N}(0)=\underline{N}(\pi)=\bar{N}(0)=\bar{N}(\pi)=0 .
\end{aligned}
$$

Recall that $v(x)=\sin (x)$ is an eigenvector corresponding to the principal eigenvalue $\lambda_{0}=-d_{1}-\delta-\beta(0)+$ $2 e^{-\gamma \tau-d_{2} \tau} \beta(0)$ of (4.3). For the proof of existence of a positive stationary solution, we need the following additional conditions:

$$
\beta^{\star}:=\sup _{s \geq 0}[\beta(s) s]<+\infty
$$

and

$$
\beta_{\star}:=\inf _{s \geq 0}[\beta(s) s]^{\prime}>-\infty
$$

The conditions (5.6) and (5.7) are always satisfied in the case of $\beta$ a Hill function (2.1) (see Fig. 2). We state the following lemma.

Theorem 5.1. Assume that (5.6) holds and

$$
d_{1}+\delta+\beta(0)<2 e^{-\gamma \tau-d_{2} \tau} \beta(0)
$$

Then, for sufficiently small $\epsilon>0$ and large $M>0(M>\epsilon)$, we have

$$
\underline{N}(x):=\epsilon v(x) \quad \text { and } \quad \bar{N}(x):=M v(x),
$$

with $v(x)=\sin (x), x \in(0, \pi)$, as sub-solution and super-solution of (5.1).

Proof. We have $\lambda_{0}>0$ and $v(x)=\sin (x)$. It is easy to see that $\underline{N}(x)$ and $\bar{N}(x)$ satisfy (5.4) and (5.5). Moreover, we have

$$
\begin{aligned}
H(\underline{N})(x) & =H(\epsilon v)(x) \\
& =d_{1} \frac{\mathrm{d}^{2}(\epsilon v(x))}{\mathrm{d} x^{2}}-(\delta+\beta(\epsilon v(x))) \epsilon v(x)+2 e^{-\gamma \tau} \int_{0}^{\pi} \Gamma_{2}(\tau, x, s) \beta(\epsilon v(s)) \epsilon v(s) \mathrm{d} s, \\
& =\epsilon\left[d_{1} \frac{\mathrm{d}^{2} v(x)}{\mathrm{d} x^{2}}-(\delta+\beta(\epsilon v(x))) v(x)+2 e^{-\gamma \tau} \int_{0}^{\pi} \Gamma_{2}(\tau, x, s) \beta(\epsilon v(s)) v(s) \mathrm{d} s\right], \\
& =\epsilon\left[d_{1} \frac{\mathrm{d}^{2} v(x)}{\mathrm{d} x^{2}}-(\delta+\beta(0)) v(x)+2 e^{-\gamma \tau} \beta(0) \int_{0}^{\pi} \Gamma_{2}(\tau, x, s) v(s) \mathrm{d} s\right.
\end{aligned}
$$




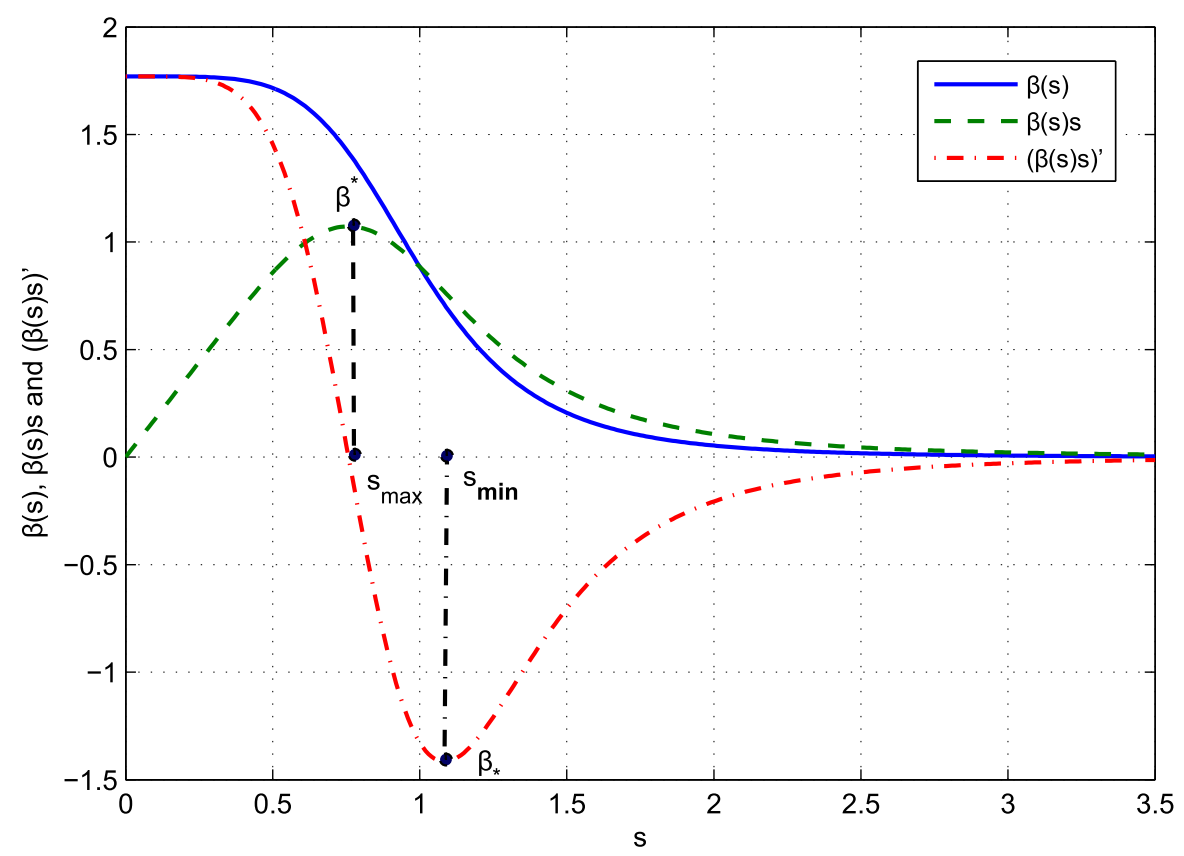

Figure 2. The Hill function $N \mapsto \beta(N)$ (see (2.1)), the function $N \mapsto \beta(N) N$ with the maximum $\beta^{\star}$ at $s_{\max }:=\theta /(r-1)^{1 / r}$ and the function $N \mapsto(\beta(N) N)^{\prime}$ with the minimum $\beta_{\star}$ at $s_{\min }=\left[(r+1) \theta^{r} /(r-1)\right]^{1 / r}$ are plotted with the parameters $r=5, \theta=1$ and $\beta_{0}=1.77$.

$$
\begin{aligned}
& \left.+(\beta(0)-\beta(\epsilon v(x))) v(x)+2 e^{-\gamma \tau} \int_{0}^{\pi} \Gamma_{2}(\tau, x, s)(\beta(\epsilon v(s))-\beta(0)) v(s) \mathrm{d} s\right], \\
= & \epsilon\left[\lambda_{0} v(x)+(\beta(0)-\beta(\epsilon v(x))) v(x)\right. \\
& \left.+2 e^{-\gamma \tau} \int_{0}^{\pi} \Gamma_{2}(\tau, x, s)(\beta(\epsilon v(s))-\beta(0)) v(s) \mathrm{d} s\right] .
\end{aligned}
$$

It is known that

$$
\int_{0}^{\pi} \Gamma_{2}(\tau, x, s) v(s) \mathrm{d} s=e^{-d_{2} \tau} v(x) .
$$

Using the fact that $\beta$ is a monotone decreasing function, we have from (5.10) that

$$
\begin{aligned}
H(\underline{N})(x) & \geq \epsilon\left[\lambda_{0} v(x)+2 e^{-\gamma \tau}(\beta(\epsilon)-\beta(0)) \int_{0}^{\pi} \Gamma_{2}(\tau, x, s) v(s) \mathrm{d} s\right], \\
& =\epsilon\left[\lambda_{0}+2 e^{-\gamma \tau-d_{2} \tau}(\beta(\epsilon)-\beta(0))\right] v(x) .
\end{aligned}
$$

Since $\lambda_{0}>0$, it follows from the continuity of $\beta$ that the inequality (5.2) holds for a sufficiently small $\epsilon>0$.

In another side, we have

$$
v^{\prime \prime}(x)=-\sin (x)=-v(x), \quad x \in(0, \pi) .
$$


Then,

$$
\begin{aligned}
H(\bar{N})(x)= & H(M v)(x), \\
= & d_{1} \frac{\mathrm{d}^{2}(M v(x))}{\mathrm{d} x^{2}}-(\delta+\beta(M v(x))) M v(x) \\
& +2 e^{-\gamma \tau} \int_{0}^{\pi} \Gamma_{2}(\tau, x, s) \beta(M v(s)) M v(s) \mathrm{d} s, \\
= & M\left[d_{1} \frac{\mathrm{d}^{2} v(x)}{\mathrm{d} x^{2}}-(\delta+\beta(M v(x))) v(x)\right. \\
& \left.+2 e^{-\gamma \tau} \int_{0}^{\pi} \Gamma_{2}(\tau, x, s) \beta(M v(s)) v(s) \mathrm{d} s\right], \\
= & M\left[-d_{1} v(x)-\delta v(x)-\beta(M v(x)) v(x)\right. \\
& \left.+2 e^{-\gamma \tau} \int_{0}^{\pi} \Gamma_{2}(\tau, x, s) \beta(M v(s)) v(s) \mathrm{d} s\right] .
\end{aligned}
$$

We need the following equality, for $x \in \mathbb{R}$,

$$
\sin (k x)=\sin (x) f_{k}(x), \quad k=1,2, \ldots,
$$

where

$$
f_{k}(x):= \begin{cases}1, & k=1, \\ 2 \cos (x), & k=2, \\ 2 \cos ^{k-1}(x)+\sum_{\ell=2}^{k-1} \cos (\ell x) \cos ^{k-1-\ell}(x), & k=3,4, \ldots\end{cases}
$$

Furthermore, we have

$$
\begin{aligned}
\int_{0}^{\pi} \Gamma_{2}(\tau, x, s) \beta(M v(s)) v(s) \mathrm{d} s & =\frac{1}{M} \int_{0}^{\pi} \Gamma_{2}(\tau, x, s) \beta(M v(s)) M v(s) \mathrm{d} s, \\
& \leq \frac{\beta^{\star}}{M} \int_{0}^{\pi} \Gamma_{2}(\tau, x, s) \mathrm{d} s, \\
& =\frac{2 \beta^{\star}}{\pi M} \int_{0}^{\pi} \sum_{k=1}^{+\infty} \sin (k s) \sin (k x) e^{-k^{2} d_{2} \tau} \mathrm{d} s, \\
& =\frac{2 \beta^{\star}}{\pi M} \sum_{k=1}^{+\infty} \sin (k x) e^{-k^{2} d_{2} \tau} \int_{0}^{\pi} \sin (k s) \mathrm{d} s, \\
& =\frac{4 \beta^{\star}}{\pi M} \sum_{k=1}^{+\infty} \sin (2 k-1) x \frac{e^{-(2 k-1)^{2} d_{2} \tau}}{2 k-1}, \\
& =\frac{4 \beta^{\star}}{\pi M} \sin (x) \sum_{k=1}^{+\infty} f_{2 k-1}(x) \frac{e^{-(2 k-1)^{2} d_{2} \tau}}{2 k-1},
\end{aligned}
$$


where

$$
f_{2 k-1}(x):=2 \cos ^{2 k-2}(x)+\sum_{\ell=2}^{2 k-2} \cos (\ell x) \cos ^{2 k-2-\ell}(x) \leq 2+\sum_{\ell=2}^{2 k-2} 1=2 k-1 .
$$

This leads to the following estimation:

$$
H(\bar{N})(x) \leq M\left[-d_{1}-\delta-\beta(M v(x))+\frac{8 \beta^{\star} e^{-\gamma \tau}}{\pi M} \sum_{k=1}^{+\infty} e^{-(2 k-1)^{2} d_{2} \tau}\right] v(x) .
$$

Hence, we obtain $H(\bar{N})(x) \leq 0$ for sufficiently large $M>0$. Consequently, $\underline{N}(x)$ and $\bar{N}(x)$ as defined in (5.9), are sub-solution and super-solution, respectively.

We are going now to prove the existence of positive solution $N(x)$ of the stationary problem (5.1). We will use the previous theorem. We can remark that for $\beta_{\star} \geq 0$, where $\beta_{\star}$ is given by (5.7), we have $[\beta(s) s]^{\prime} \geq 0$ for all $s \geq 0$ (monotone case), and for $\beta_{\star}<0$ the expression $s \mapsto[\beta(s) s]^{\prime}$ changes the sign over the interval $[0,+\infty$ ) (non-monotone case). We put

$$
\mu:=\min \left\{0, \beta_{\star}\right\} \leq 0
$$

Then, the stationary problem (5.1) is equivalent to the following system:

$$
\left\{\begin{array}{l}
-d_{1} \frac{\mathrm{d}^{2} N(x)}{\mathrm{d} x^{2}}+(\delta+\beta(0)) N(x)-2 \mu e^{-\gamma \tau} \int_{0}^{\pi} \Gamma_{2}(\tau, x, s) N(s) \mathrm{d} s \\
=(\beta(0)-\beta(N(x))) N(x)+2 e^{-\gamma \tau} \int_{0}^{\pi} \Gamma_{2}(\tau, x, s) h(N(s)) \mathrm{d} s, \\
N(0)=N(\pi)=0,
\end{array}\right.
$$

where

$$
h(N):=\beta(N) N-\mu N, \quad N \in[0,+\infty) .
$$

This transformation leads to have $h^{\prime}(N) \geq 0$ for all $N \geq 0$. We consider the following differential operator:

$$
\mathcal{L}: u \in C^{2}(0, \pi) \cap C[0, \pi] \mapsto \mathcal{L} u=-d_{1} \frac{\mathrm{d}^{2} u}{\mathrm{~d} x^{2}}+b_{1} u \in C[0, \pi]
$$

with $d_{1}, b_{1}>0$ and we define $\mathcal{K}: C[0, \pi] \rightarrow C^{2}(0, \pi) \cap C[0, \pi]$ by

$$
(\mathcal{K} u)(x)=b_{2} \int_{0}^{\pi} \Gamma_{2}(\tau, x, s) u(s) \mathrm{d} s
$$

with $b_{2} \geq 0$. The following lemma was proved in [14].

Lemma 5.2. If

$$
\frac{4 b_{2}}{\pi\left(d_{1}+b_{1}\right)} \frac{1}{e^{d_{2} \tau}-1}<1
$$


and

$$
\frac{8 b_{2} \sinh ^{4}\left(\frac{\sqrt{b_{1}} \pi}{2 \sqrt{d_{1}}}\right)}{\pi d_{1}\left(e^{d_{2} \tau}-1\right)\left(\frac{\sqrt{b_{1}}}{\sqrt{d_{1}}}\right)^{3} \sinh \left(\frac{\sqrt{b_{1}} \pi}{\sqrt{d_{1}}}\right)}<1,
$$

hold simultaneously, then $(\mathcal{L}+\mathcal{K})^{-1}$ is positive.

Remark 5.3. If $\mu=0$, then $\mathcal{L}+\mathcal{K}=\mathcal{L}$ and clearly $\mathcal{L}^{-1}$ exists and is positive [14].

Using the last lemma, we prove the following proposition:

Proposition 5.4. Let $b_{1}=\delta+\beta(0)$ and $b_{2}=-2 \mu e^{-\gamma \tau}$. Assume that (5.6), (5.7), (5.13) and (5.14) hold, and the inequality (5.8):

$$
d_{1}+\delta+\beta(0)<2 e^{-\gamma \tau-d_{2} \tau} \beta(0)
$$

Then, there exists a positive stationary solution $N(x)$ of problem (5.1).

Proof. From Theorem 5.1, the functions $\underline{N}(x)$ and $\bar{N}(x)$ defined in (5.9) are sub-solution and super-solution, respectively. Let consider the operators $\mathcal{L}$ and $\mathcal{K}$ defined as before with $b_{1}=\delta+\beta(0)$ and $b_{2}=-2 \mu e^{-\gamma \tau}$. We define the nonlinear operator $\mathcal{P}: C[0, \pi] \rightarrow C^{2}(0, \pi) \cap C[0, \pi]$ by

$$
(\mathcal{P} u)(x)=(\mathcal{L}+\mathcal{K})^{-1}\left((\beta(0)-\beta(u)) u+2 e^{-\gamma \tau} \int_{0}^{\pi} \Gamma_{2}(\tau, x, s) h(u(s)) \mathrm{d} s\right) .
$$

Recall that $u \mapsto \beta(u)$ is a decreasing function for $u \geq 0$. So, $u \mapsto(\beta(0)-\beta(u)) u$ is an increasing function for $u \geq 0$. Furthermore, $u \mapsto h(u)$ is also an increasing function for $u \geq 0$. We know also from (2.6) that $\Gamma_{2}$ is positive. Then, we can say that the operator $\mathcal{Q}: C[0, \pi] \rightarrow C[0, \pi]$ given by

$$
(\mathcal{Q} u)(x)=(\beta(0)-\beta(u)) u+2 e^{-\gamma \tau} \int_{0}^{\pi} \Gamma_{2}(\tau, x, s) h(u(s)) \mathrm{d} s,
$$

is positive and strongly monotone. Moreover, $(\mathcal{L}+\mathcal{K})^{-1}$ is positive (see Lem. 5.2). This implies that $\mathcal{P}$ is positive and strongly monotone. Let denote by $\hat{K}$, the constant function on $[0, \pi]$ taking the constant value $K$. Then, $\mathcal{P}$ is strongly increasing on the interval $[\hat{0}, \hat{M}]$, where $M$ is given by Theorem 5.1. By using a standard argument of sub- and super-solutions (see [23]) and Theorem 5.1, we conclude that there exists a positive stationary solution $N^{\star}(x)$ of problem (5.1), which satisfies

$$
0<\underline{N}(x) \leq N^{\star}(x) \leq \bar{N}(x), \quad \text { for all }(0, \pi)
$$

Remark 5.5. The conditions (5.13) and (5.14) can hold simultaneously. Indeed, for the Hill function $\beta$ given by (2.1), the only parameter in (5.13) and (5.14) that depends on the sensitivity $r$ is $\mu$ and we can see that $\lim _{r \rightarrow 1^{+}} \mu(r)=0$. Consequently, (5.13) and (5.14) hold simultaneously for $r$ near 1 , from above.

Let now prove that no positive solution of (5.1) exists beyond $\bar{N}$ and then all the solutions are in the interval $[0, \bar{N}]$. 
Theorem 5.6. Suppose that the assumptions of Theorem 5.1 hold. Then, the problem (5.1) has no positive solution beyond the super-solution $x \mapsto \bar{N}(x)$.

Proof. Because $\epsilon>0$ is arbitrary (see Thm. 5.1), we know that (5.1) has a positive solution in the order interval $[0, \bar{N}]$. Let prove that no positive solution exists beyond $\bar{N}$. Assume by contradiction that a positive solution $N$ of (5.1) exists with

$$
\max _{s \in[0, \pi]} N(s)>\bar{N}(x)=M \sin (x), \quad x \in(0, \pi) .
$$

So, there exists $x_{0} \in(0, \pi)$ such that $N\left(x_{0}\right)=\max _{x \in[0, \pi]} N(x)$. Obviously, we can choose a large $M$ to have

$$
M \delta \sin \left(x_{0}\right)-2 e^{-\gamma \tau} \beta^{\star} \int_{0}^{\pi} \Gamma_{2}\left(\tau, x_{0}, s\right) \mathrm{d} s>0 .
$$

Then, by using the hypothesis (5.6), we obtain

$$
\begin{aligned}
& -d_{1} \frac{\mathrm{d}^{2} N\left(x_{0}\right)}{\mathrm{d} x^{2}}+\left(\delta+\beta\left(N\left(x_{0}\right)\right)\right) N\left(x_{0}\right)-2 e^{-\gamma \tau} \int_{0}^{\pi} \Gamma_{2}\left(\tau, x_{0}, s\right) \beta(N(s)) N(s) \mathrm{d} s \\
& >M \delta \sin \left(x_{0}\right)-2 e^{-\gamma \tau} \beta^{\star} \int_{0}^{\pi} \Gamma_{2}\left(\tau, x_{0}, s\right) \mathrm{d} s>0 .
\end{aligned}
$$

This leads to a contradiction.

For the uniqueness of the stationary solution of problem (5.1), we make the following additional assumption.

$$
u \mapsto\left[\left(2 e^{-\gamma \tau-d_{2} \tau}-1\right) \beta(u)-\left(d_{1}+\delta\right)\right] u \text { is nondecreasing for } u \geq 0 .
$$

Under the assumption (5.15), we prove the following theorem on the uniqueness of the stationary solution.

Theorem 5.7. Suppose that the assumptions of Theorem 5.1 and (5.15) hold. Then, problem (5.1) has at most one positive stationary solution.

Proof. Suppose that there exist two nonnegative stationary solutions $N_{1}(x)$ and $N_{2}(x)$ for problem (5.1). From Theorem 5.1, we have that $\epsilon v(x)=\underline{N}(x) \leq N_{1}(x), N_{2}(x) \leq \bar{N}(x)=M v(x)$ for all $x \in[0, \pi]$. Hence, we obtain

$$
N_{1}(x) \geq \epsilon v(x)=\frac{\epsilon}{M} M v(x) \geq \frac{\epsilon}{M} N_{2}(x), \quad x \in[0, \pi] .
$$

Let $\xi:=\sup \left\{c \in \mathbb{R}^{+} \mid N_{1}(x) \geq c N_{2}(x)\right.$, for all $\left.x \in[0, \pi]\right\}$. From (5.16), we see that $\xi>0$. Suppose that $\xi \in(0,1)$ and find a contradiction. Recall that $v(x)=\sin (x)$ and the inequality (4.8). Then,

$$
\int_{0}^{\pi} v(x) d_{1} \frac{\mathrm{d}^{2} N_{1}(x)}{\mathrm{d} x^{2}} \mathrm{~d} x=\int_{0}^{\pi} d_{1} \frac{\mathrm{d}^{2} v(x)}{\mathrm{d} x^{2}} N_{1}(x) \mathrm{d} x=-\int_{0}^{\pi} v(x) d_{1} N_{1}(x) \mathrm{d} x .
$$

Hence, we have from (5.1) and (5.15) that

$$
\begin{aligned}
0= & \int_{0}^{\pi} v(x)\left[d_{1} \frac{\mathrm{d}^{2} N_{1}(x)}{\mathrm{d} x^{2}}-\left(\delta+\beta\left(N_{1}(x)\right)\right) N_{1}(x)\right. \\
& \left.+2 e^{-\gamma \tau} \int_{0}^{\pi} \Gamma_{2}(\tau, x, s) \beta\left(N_{1}(s)\right) N_{1}(s) \mathrm{d} s\right] \mathrm{d} x,
\end{aligned}
$$




$$
\begin{aligned}
& =\int_{0}^{\pi} v(x)\left[-\left(d_{1}+\delta+\beta\left(N_{1}(x)\right)\right) N_{1}(x)+2 e^{-\gamma \tau-d_{2} \tau} \beta\left(N_{1}(x)\right) N_{1}(x)\right] \mathrm{d} x, \\
& =\int_{0}^{\pi} v(x)\left[\left(2 e^{-\gamma \tau-d_{2} \tau}-1\right) \beta\left(N_{1}(x)\right)-\left(d_{1}+\delta\right)\right] N_{1}(x) \mathrm{d} x \\
& \geq \int_{0}^{\pi} v(x)\left[\left(2 e^{-\gamma \tau-d_{2} \tau}-1\right) \beta\left(\xi N_{2}(x)\right)-\left(d_{1}+\delta\right)\right] \xi N_{2}(x) \mathrm{d} x .
\end{aligned}
$$

From (5.8), it follows that $2 e^{-\gamma \tau-d_{2} \tau}-1>0$. Hence, we obtain

$$
\begin{aligned}
0> & \int_{0}^{\pi} v(x)\left[\left(2 e^{-\gamma \tau-d_{2} \tau}-1\right) \beta\left(N_{2}(x)\right)-\left(d_{1}+\delta\right)\right] \xi N_{2}(x) \mathrm{d} x, \\
= & \xi \int_{0}^{\pi} v(x)\left[d_{1} \frac{\mathrm{d}^{2} N_{2}(x)}{\mathrm{d} x^{2}}-\left(\delta+\beta\left(N_{2}(x)\right)\right) N_{2}(x)\right. \\
& \left.+2 e^{-\gamma \tau} \int_{0}^{\pi} \Gamma_{2}(\tau, x, s) \beta\left(N_{2}(s)\right) N_{2}(s) \mathrm{d} s\right] \mathrm{d} x, \\
= & \xi \cdot 0=0 .
\end{aligned}
$$

This gives a contradiction. Hence, $\xi \geq 1$ and thus, $N_{1}(x) \geq \xi N_{2}(x) \geq N_{2}(x)$, for all $x \in[0, \pi]$. Similarly, by exchanging the roles of $N_{1}(x)$ and $N_{2}(x)$, we can show that $N_{2}(x) \geq N_{1}(x)$, for all $x \in[0, \pi]$. Consequently, $N_{1}(x)=N_{2}(x)$, for all $x \in[0, \pi]$. The proof is complete.

\section{UNIFORM PERSISTENCE}

We prove the uniform persistence of the delayed reaction-diffusion equation (2.7) under the condition (5.8) (see [12]):

$$
d_{1}+\delta+\beta(0)<2 e^{-\gamma \tau-d_{2} \tau} \beta(0)
$$

Let $D_{0}:=\left\{\phi \in \mathbb{Y}^{+}: \phi(\cdot)(x)>0\right.$, for some $\left.x \in(0, \pi)\right\}$. The uniform (strong) persistence in $D_{0}$ means that there exists a positive constant $\epsilon>0$ such that

$$
\liminf _{t \rightarrow+\infty}\|N(t, \cdot)\|_{\mathbb{X}} \geq \epsilon
$$

for any initial condition $\phi \in D_{0}$. We first prove the following lemma (Figs. 3 and 4):

Lemma 6.1. Suppose that (5.8) holds. Then, the reaction-diffusion system (2.7) is uniformly weakly persistent in $D_{0}$, that is, there exists a positive constant $\epsilon>0$ such that

$$
\limsup _{t \rightarrow+\infty}\|N(t, \cdot)\|_{\mathbb{X}} \geq \epsilon,
$$

for any initial condition $\phi \in D_{0}$.

Proof. From (5.8), we have

$$
\frac{2 \beta(0) e^{-\gamma \tau-d_{2} \tau}}{d_{1}+\delta+\beta(0)}>1 .
$$



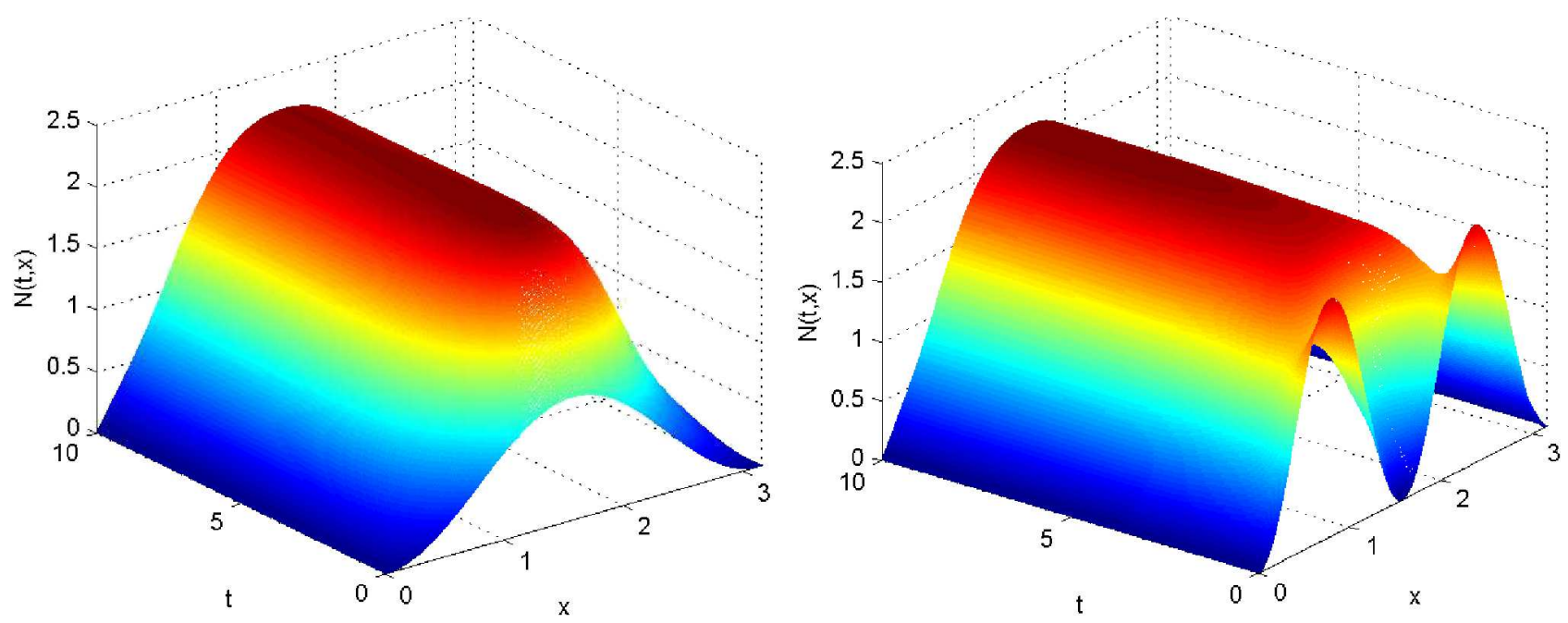

FiguRE 3. The positive stationary solution is drawn when it is stable. The function $\beta$ is taken as a Hill function. The parameters are : $\delta=0.05, \beta_{0}=1.77, \theta=1, \gamma=0.2, \tau=0.5, r=5$, $d_{1}=0.1$ and $d_{2}=0.1$. The initial conditions are $N(\theta, x)=(1-\cos (2 x)) / 2$ in the left and $N(\theta, x)=1-\cos (4 x)$ in the right for $x \in[0, \pi]$ and $\theta \in[-\tau, 0]$.
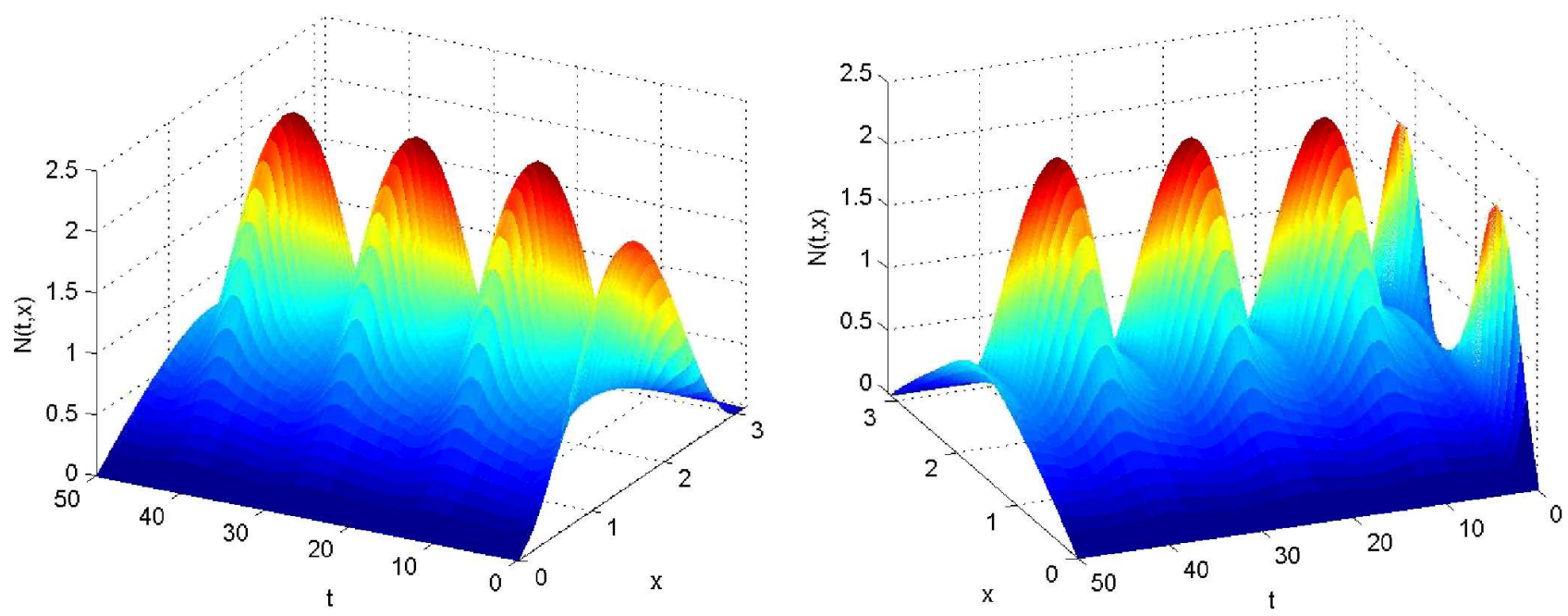

Figure 4. A special case is carried out to show by simulations that a possible Hopf bifurcation can occur. Clearly the solutions oscillate periodically around the positive nonuniform stationary solution. The function $\beta$ is taken as a Hill function. The parameters are $: \delta=0.05, \beta_{0}=1.77$, $\theta=1, \gamma=0.2, \tau=1.7, r=5, d_{1}=0.1$ and $d_{2}=0.1$. The initial conditions are $N(\theta, x)=$ $(1-\cos (2 x)) / 2$ in the left and $N(\theta, x)=1-\cos (4 x)$ in the right, for $x \in[0, \pi]$ and $\theta \in[-\tau, 0]$.

Hence, from the continuity, there exist sufficiently small $\epsilon>0$ such that

$$
\mathcal{R}_{\epsilon, \lambda}:=\frac{2 \beta(\epsilon) e^{-\gamma \tau-d_{2} \tau}}{\lambda+d_{1}+\delta+\beta(0)}>1, \quad \text { for } \lambda>0
$$


Suppose that (6.1) does not hold. Then, there exists a sufficiently large $T>0$ such that

$$
N(t, x)<\epsilon, \quad \text { for all } t \geq T \text { and } x \in[0, \pi] .
$$

In what follows, without loss of generality, we can assume that $T=0$ since we can choose $N_{T}(\theta)(x):=N(T+$ $\theta, x), \theta \in[-\tau, 0]$, as the new initial condition in $D_{0}$. Let $t \geq \tau$. As $\beta$ is a decreasing function, we have from $(2.7)$

$$
\begin{aligned}
\frac{\partial N(t, x)}{\partial t} \geq & d_{1} \frac{\partial^{2} N(t, x)}{\partial x^{2}}-(\delta+\beta(0)) N(t, x) \\
& +2 e^{-\gamma \tau} \int_{0}^{\pi} \Gamma_{2}(\tau, x, s) \beta(N(t-\tau, s)) N(t-\tau, s) \mathrm{d} s .
\end{aligned}
$$

Hence, from the comparison principle, we obtain

$$
\begin{aligned}
N(t, x) \geq & N(\tau, x) e^{-(\delta+\beta(0))(t-\tau)}+\int_{\tau}^{t} e^{-(\delta+\beta(0))(t-u)}\left[d_{1} \frac{\partial^{2} N(u, x)}{\partial x^{2}}\right. \\
& \left.+2 e^{-\gamma \tau} \int_{0}^{\pi} \Gamma_{2}(\tau, x, y) \beta(N(u-\tau, y)) N(u-\tau, y) \mathrm{d} y\right] \mathrm{d} u \\
\geq & \int_{\tau}^{t} e^{-(\delta+\beta(0))(t-u)}\left[d_{1} \frac{\partial^{2} N(u, x)}{\partial x^{2}}\right. \\
& \left.+2 e^{-\gamma \tau} \int_{0}^{\pi} \Gamma_{2}(\tau, x, y) \beta(N(u-\tau, y)) N(u-\tau, y) \mathrm{d} y\right] \mathrm{d} u \\
\geq & \int_{0}^{t-\tau} e^{-(\delta+\beta(0))(t-\tau-\sigma)}\left[d_{1} \frac{\partial^{2} N(\sigma+\tau, x)}{\partial x^{2}}\right. \\
& \left.+2 \beta(\epsilon) e^{-\gamma \tau} \int_{0}^{\pi} \Gamma_{2}(\tau, x, y) N(\sigma, y) \mathrm{d} y\right] \mathrm{d} \sigma .
\end{aligned}
$$

Hence, by changing $t \rightarrow t+\tau$, we get

$$
N(t+\tau, x) \geq \int_{0}^{t} e^{-(\delta+\beta(0))(t-\sigma)}\left[d_{1} \frac{\partial^{2} N(\sigma+\tau, x)}{\partial x^{2}}+2 \beta(\epsilon) e^{-\gamma \tau} \int_{0}^{\pi} \Gamma_{2}(\tau, x, y) N(\sigma, y) \mathrm{d} y\right] \mathrm{d} \sigma .
$$

Multiplying by $v(x)=\sin (x)$ in both sides and integrating, we have

$$
\begin{aligned}
\int_{0}^{\pi} v(x) N(t+\tau, x) \mathrm{d} x \geq & \int_{0}^{\pi} v(x) \int_{0}^{t} e^{-(\delta+\beta(0))(t-\sigma)}\left[d_{1} \frac{\partial^{2} N(\sigma+\tau, x)}{\partial x^{2}}\right. \\
& \left.+2 \beta(\epsilon) e^{-\gamma \tau} \int_{0}^{\pi} \Gamma_{2}(\tau, x, y) N(\sigma, y) \mathrm{d} y\right] \mathrm{d} \sigma \mathrm{d} x \\
= & \int_{0}^{\pi} v(x) \int_{0}^{t} e^{-(\delta+\beta(0))(t-\sigma)}\left[-d_{1} N(\sigma+\tau, x)+2 \beta(\epsilon) e^{-\gamma \tau-d_{2} \tau} N(\sigma, x)\right] \mathrm{d} \sigma \mathrm{d} x .
\end{aligned}
$$


Taking the Laplace transform of both sides for $\lambda>0$, we obtain

$$
\begin{aligned}
\int_{0}^{+\infty} & e^{-\lambda t} \int_{0}^{\pi} v(x) N(t+\tau, x) \mathrm{d} x \mathrm{~d} t \\
\geq & -d_{1} \int_{0}^{+\infty} e^{-\lambda t} \int_{0}^{\pi} v(x) \int_{0}^{t} e^{-(\delta+\beta(0))(t-\sigma)} N(\sigma+\tau, x) \mathrm{d} \sigma \mathrm{d} x \mathrm{~d} t \\
& +2 \beta(\epsilon) e^{-\gamma \tau-d_{2} \tau} \int_{0}^{+\infty} e^{-\lambda t} \int_{0}^{\pi} v(x) \int_{0}^{t} e^{-(\delta+\beta(0))(t-\sigma)} N(\sigma, x) \mathrm{d} \sigma \mathrm{d} x \mathrm{~d} t \\
= & -\frac{d_{1}}{\lambda+\delta+\beta(0)} \int_{0}^{+\infty} e^{-\lambda t} \int_{0}^{\pi} v(x) N(t+\tau, x) \mathrm{d} x \mathrm{~d} t \\
& +\frac{2 \beta(\epsilon) e^{-\gamma \tau-d_{2} \tau}}{\lambda+\delta+\beta(0)} \int_{0}^{+\infty} e^{-\lambda t} \int_{0}^{\pi} v(x) N(t, x) \mathrm{d} x \mathrm{~d} t .
\end{aligned}
$$

Hence, we have

$$
\begin{aligned}
& \int_{0}^{+\infty} e^{-\lambda t} \int_{0}^{\pi} v(x) N(t, x) \mathrm{d} x \mathrm{~d} t \\
& \quad \leq \frac{\lambda+d_{1}+\delta+\beta(0)}{2 \beta(\epsilon) e^{-\gamma \tau-d_{2} \tau}} \int_{0}^{+\infty} e^{-\lambda t} \int_{0}^{\pi} v(x) N(t+\tau, x) \mathrm{d} x \mathrm{~d} t \\
& \quad=\frac{1}{\mathcal{R}_{\epsilon, \lambda}} \int_{0}^{+\infty} e^{-\lambda t} \int_{0}^{\pi} v(x) N(t+\tau, x) \mathrm{d} x \mathrm{~d} t
\end{aligned}
$$

In a completely similar manner, we can prove for any $n \in \mathbb{N}$ that

$$
\begin{aligned}
& \int_{0}^{+\infty} e^{-\lambda t} \int_{0}^{\pi} v(x) N(t+(n-1) \tau, x) \mathrm{d} x \mathrm{~d} t \\
& \quad \leq \frac{1}{\mathcal{R}_{\epsilon, \lambda}} \int_{0}^{+\infty} e^{-\lambda t} \int_{0}^{\pi} v(x) N(t+n \tau, x) \mathrm{d} x \mathrm{~d} t .
\end{aligned}
$$

Hence, from the boundedness of $N$, we have

$$
\begin{aligned}
\int_{0}^{+\infty} e^{-\lambda t} \int_{0}^{\pi} v(x) N(t, x) \mathrm{d} x \mathrm{~d} t & \leq \frac{1}{\left(\mathcal{R}_{\epsilon, \lambda}\right)^{n}} \int_{0}^{+\infty} e^{-\lambda t} \int_{0}^{\pi} v(x) N(t+n \tau, x) \mathrm{d} x \mathrm{~d} t \\
& \leq \frac{2 \epsilon}{\lambda} \frac{1}{\left(\mathcal{R}_{\epsilon, \lambda}\right)^{n}} \rightarrow 0 \quad \text { as } n \rightarrow+\infty
\end{aligned}
$$

This implies that $N \equiv 0$. This contradicts the positivity of $N$ (see Rem. 2.3). Therefore, (6.1) holds and the proof is complete.

Since the set $\{0\}$ of the trivial steady state is a singleton in $\mathbb{X}^{+}$, it is compact. Hence, it follows from ([12], Thm. 2) that the uniform weak persistence implies the uniform (strong) persistence. Consequently, we have the following theorem on the uniform persistence of problem (2.7) (Figs. 3 and 4).

Theorem 6.2. Suppose that (5.8) holds. Then, the system (2.7) is uniformly persistent in $D_{0}$, that is, there exists a positive constant $\epsilon>0$ such that

$$
\liminf _{t \rightarrow+\infty}\|N(t, \cdot)\|_{\mathbb{X}} \geq \epsilon
$$

for any initial condition $\phi \in D_{0}$. 


\section{REFERENCES}

[1] M. Adimy and F. Crauste, Global stability of a partial differential equation with distributed delay due to cellular replication. Nonlinear Anal.: Theory Meth. Appl. 54 (2003) 1469-1491.

[2] M. Adimy, F. Crauste and S. Ruan, A mathematical study of the hematopoiesis process with applications to chronic myelogenous leukemia. SIAM J. Appl. Math. 65 (2005) 1328-1352.

[3] M. Adimy, A. Chekroun and T.-M. Touaoula, Age-structured and delay differential-difference model of hematopoietic stem cell dynamics. Discrete Contin. Dyn. Syst. Ser. B 20 (2015) 2765-2791.

[4] M. Adimy, A. Chekroun and B. Kazmierczak, Traveling waves in a coupled reaction-diffusion and difference model of hematopoiesis. J. Diff. Eq. 262 (2017) 4085-4128.

[5] S. Bernard, J. Bélair and M.C. Mackey, Oscillations in cyclical neutropenia: new evidence based on mathematical modeling. J. Theor. Biol. 223 (2003) 283-298.

[6] S. Bernard, J. Bélair and M.C. Mackey, Bifurcations in a white-blood-cell production model. C.R. Biol. 327 (2004) 201-210.

[7] N. Bessonov, F. Crauste, I. Demin and V. Volpert, Dynamics of erythroid progenitors and erythroleukemia. Math. Model. Nat. Phenom. 4 (2009) 210-232.

[8] F.J. Burns and I.F. Tannock, On the existence of a $\mathrm{G}_{0}$-phase in the cell cycle. Cell Prolif. 3 (1970) 321-334.

[9] S. Chen and J. Shi, Stability and Hopf bifurcation in a diffusive logistic population model with nonlocal delay effect. J. Diff. Eq. 253 (2012) 3440-3470.

[10] A. Ducrot and V. Volpert, On a model of leukemia development with a spatial cell distribution. Math. Model. Nat. Phenom. 2 (2007) 101-120.

[11] T. Faria, Stability and bifurcation for a delayed predator-prey model and the effect of diffusion. J. Math. Anal. Appl. 254 (2001) 433-463.

[12] H.I. Freedman and P. Moson, Persistence definitions and their connections. Proc. Am. Math. Soc. 109 (1990) $1025-1033$.

[13] S.-A. Gourley and J. Wu, Delayed non-local diffusive systems in biological invasion and disease spread. Nonlinear Dynamics and Evolution Equations. Amer. Math. Soc. 48 (2006) 137-200.

[14] Z. Guo, Z.-C. Yang and X. Zou, Existence and uniqueness of positive solution to a non-local differential equation with homogeneous Dirichlet boundary condition - a non-monotone case. Commun. Pure Appl. Anal. 11 (2012) 1825-1838.

[15] G.-P. Hu and W.-T. Li, Hopf bifurcation analysis for a delayed predator-prey system with diffusion effects. Nonlinear Anal.: Real World Appl. 11 (2010) 819-826.

[16] T. Kuniya and J. Wang, Lyapunov functions and global stability for a spatially diffusive SIR epidemic model. Appl. Anal. 96 (2016) 1935-1960.

[17] L. Lajtha, On DNA labeling in the study of the dynamics of bone marrow cell populations, in The Kinetics of Cellular Proliferation, F. Stohlman, Jr., ed. Grune and Stratton, New York (1959) 173-182.

[18] Z. Ling and Z. Lin, Traveling wavefront in a hematopoiesis model with time delay. Appl. Math. Lett. 23 (2010) $426-431$.

[19] Z.-P. Ma, W.-T. Li and X.-P. Yan, Stability and Hopf bifurcation for a three-species food chain model with time delay and spatial diffusion. Appl. Math. Comput. 219 (2012) 2713-2731.

[20] M.C. Mackey, Unified hypothesis for the origin of aplastic anemia and periodic hematopoiesis. Blood 51 (1978) 941-956.

[21] M.C. Mackey, C. Ou, L. Pujo-Menjouet and J. Wu, Periodic oscillations of blood cell populations in chronic myelogenous leukemia. SIAM J. Math. Anal. 38 (2006) 166-187.

[22] R.H. Martin and H.L. Smith, Abstract functional-differential equations and reaction-diffusion systems. Trans. Am. Math. Soc. 321 (1990) 1-44.

[23] C.V. Pao, Nonlinear Parabolic and Elliptic Equations. Springer, New York (1992).

[24] L. Pujo-Menjouet and M.C. Mackey, Contribution to the study of periodic chronic myelogenous leukemia. C.R. Biol. 327 (2004) 235-244.

[25] L. Pujo-Menjouet, S. Bernard and M. Mackey, Long period oscillations in a $\mathrm{G}_{0}$ model of hematopoietic stem cells. SIAM J. Appl. Dyn. Syst. 4 (2005) 312-332.

[26] H.L. Smith, Monotone Dynamical Systems: An Introduction to the Theory of Competitive and Cooperative Systems. Mathematical Surveys and Monographs. 41, AMS, Providence, RI (1995).

[27] J.W.-H. So, J. Wu and X. Zou, A reaction-diffusion model for a single species with age structure. I. Travelling wavefronts on unbounded domains. Proc. R. Soc. Lond. A 457 (2001) 1841-1853.

[28] Y. Su, J. Wei and J. Shi, Hopf bifurcations in a reaction-diffusion population model with delay effect. J. Diff. Eq. 247 (2009) $1156-1184$.

[29] H.R. Thieme and X.-Q. Zhao, A non-local delayed and diffusive predator-prey model. Nonlinear Anal.: Real World Appl. 2 (2001) 145-160.

[30] J.A. Walker, Dynamical Systems and Evolution Equations: Theory and Applications. Plenum Press, New York (1980).

[31] X. Wang and Z. Li, Dynamics for a type of general reaction-diffusion model. Nonlinear Anal.: Theory Meth. Appl. 67 (2007) 2699-2711.

[32] J. Wang, J. Yang and T. Kuniya, Dynamics of a PDE viral infection model incorporating cell-to-cell transmission. J. Math. Anal. Appl. 444 (2016) 1542-1564.

[33] I.L. Weissman, Stem cells: units of development, units of regeneration, and units in evolution. Cell 100 (2000) $157-168$. 
[34] J. Wu, Theory and Applications of Partial Functional Differential Equations. Applied Math. Sci. Vol 119, Springer, New York (1996).

[35] D. Xu and X.-Q. Zhao, A nonlocal reaction-diffusion population model with stage structure. Can. Appl. Math. Q. 11 (2003) 303-319.

[36] X.-P. Yan, Stability and Hopf bifurcation for a delayed prey-predator system with diffusion effects. Appl. Math. Comput. 192 (2007) 552-566.

[37] X.-Q. Zhao, Dynamical Systems in Population Biology. Springer, New York (2003).

[38] X.-Q. Zhao, Global attractivity in a class of nonmonotone reaction-diffusion equations with time delay. Can. Appl. Math. Q. 17 (2009) 271-281. 